\title{
Estimation of Combustion Phasing Using the Combustion Net Torque Method ${ }^{\star}$
}

\author{
Mikael Thor, Bo Egardt, Tomas McKelvey, Ingemar Andersson \\ Department of Signals and Systems, Chalmers University of \\ Technology, SE-412 96 Göteborg, Sweden \\ $\{$ mikael.thor,egardt,mckelvey,ia\}@chalmers.se
}

\begin{abstract}
A new and computationally efficient method, called the combustion net torque method, for estimating combustion phasing in an internal combustion engine using crankshaft torque data is presented. The work is motivated by the need of such an estimation method for real-time implementation of a torque based closed-loop combustion phasing control system. The ideal properties of the proposed method, based on torque calculated from cylinder pressure, are studied using simulations and experimental data from both a spark ignited and a compression ignited engine. A relation to $50 \%$ burned mass fraction, the most common measure of combustion phasing, is established and the robustness of the method is investigated. The results show that the ideal estimation accuracy of the proposed method is high, especially around the combustion phasing position where maximum engine efficiency is achieved. Also, the computational complexity of the combustion net torque method is significantly lower compared to other existing techniques for torque domain combustion phasing estimation.
\end{abstract}

Keywords: Internal combustion engines, Engine control, Engine management

\section{INTRODUCTION}

The environmental influence of internal combustion engines is a well known problem in today's society and it motivates the development of more efficient and less polluting engines. To find engines that meet strict future emission legislations, the use of more advanced combustion concepts is necessary. These combustion concepts benefit significantly from improved combustion control and the interest in closed-loop and adaptive combustion control systems, as opposed to traditional pre-calibrated systems for open-loop combustion control, has increased in recent years. However, any closed-loop combustion control system relies on accurate information from the combustion process and many different sensor configurations are being considered for the gathering of this information. The range of sensors span from devices located inside the cylinder, measuring e.g. pressure or ion current, to engine block accelerometers, the crankshaft torque sensors that this work focuses on, or sensors measuring the angular velocity of the flywheel. Also, a variety of exhaust gas sensors exist.

Once the sensor signals are available they need to be processed and analyzed in order to provide the control system with useful information. Methods for combustion analysis have a rich history in the literature and a vast majority of these methods are based on cylinder pressure measurements. The most common of these cylinder pressure based methods for describing the characteristics of the combustion process is the burned mass fraction, see Heywood (1988). The burned mass fraction provides, amongst other combustion properties, a measure of combustion phasing, i.e. the timing of the combustion. This measure is usually

* This work is financed by the Combustion Engine Research Center (CERC) at Chalmers University of Technology. defined as the position where half of the injected fuel mass has burned and is referred to as $50 \%$ burned mass fraction. Combustion phasing is an important combustion property as it has a significant impact not only on engine efficiency but also on engine out emissions. Hence, controlling the combustion phasing in order to counteract effects such as component wear, manufacturing variations, or varying fuel quality is a big challenge for future engine control systems.

In an engine control system that is based on instantaneous measurements of crankshaft torque it is not possible to directly calculate the burned mass fraction. The reason is that, even though it is possible to separate the measured crankshaft torque into cylinder individual torque contributions, the transformation from torque to cylinder pressure meets difficulties rooted in the geometry of the crank-slider mechanism. A method for torque domain combustion phasing estimation is therefore needed in order to enable torque based closed-loop combustion phasing control. Such a method, called torque ratio, was developed in Andersson and McKelvey (2004b) and is closely related to pressure ratio that was introduced in Matekunas (1986). A downside with the torque ratio method is that, for each cylinder, a nonlinear parameter estimation problem needs to be solved for each engine cycle. As the shape of the combustion process gets more complex, using e.g. multiple fuel injections in a diesel engine, the dimension of this estimation problem increases, see Thor et al. (2009). The computational demand of this method may therefore be a burden in real-time implementations.

This paper proposes a new and computationally efficient method, the combustion net torque method, for $50 \%$ burned mass fraction estimation from crankshaft torque measurements. Its intended application is a system 
for torque based closed-loop combustion control in an internal combustion engine. The work investigates the ideal properties of the method using torque traces calculated from cylinder pressure. A relation between the combustion net torque and $50 \%$ burned mass fraction is established based on simulations, using a simple cylinder pressure simulation model, and experimental cylinder pressure data, collected from both a spark ignited and a compression ignited engine. The experimental data is also used to assess the ideal estimation accuracy of the proposed method in a real engine.

\section{BURNED MASS FRACTION CALCULATION AND CYLINDER PRESSURE SIMULATION}

The burned mass fraction is a description of how the combustion, i.e. the process of transforming the chemical energy in the fuel into heat, evolves over time. There exist numerous methods for estimation of burned mass fraction, the first introduced in Rassweiler and Withrow (1938). Another approach, taken here, is to base the burned mass fraction estimation on the first law of thermodynamics using a single-zone heat release model similar to the one described in Gatowski et al. (1984). However, some simplifications have been made to this model, e.g. neglection of mass flows, heat transfer, and crevice effects. The result is often referred to as net heat release, see e.g. Heywood (1988). This model describes the rate with which the chemical energy, $Q(\theta)$, is released as

$$
\frac{d Q(\theta)}{d \theta}=\frac{\gamma}{\gamma-1} p(\theta) \frac{d V(\theta)}{d \theta}+\frac{1}{\gamma-1} V(\theta) \frac{d p(\theta)}{d \theta}
$$

Here, $\gamma$ is the ratio of specific heats, $p(\theta)$ is the cylinder pressure, $V(\theta)$ is the cylinder volume, and $\theta$ is the angle reference expressed in crank angle degrees (CAD). Based on the released chemical energy, the burned mass fraction, $x_{b}(\theta)$, is estimated according to

$$
x_{b}(\theta)=\frac{Q(\theta)}{Q_{t o t}}
$$

where $Q_{t o t}$, the total amount of energy released by the combustion, is described by

$$
Q_{\text {tot }}=\max _{\theta} Q(\theta)
$$

Finally, as a combustion phasing measure, the $50 \%$ burned mass fraction position, $\theta_{x_{b} 50}$, is defined as

$$
x_{b}\left(\theta_{x_{b} 50}\right)=0.5
$$

The burned mass fraction trace, and hence also the combustion process, is often characterized using two burn angles, $\theta_{d}$ and $\theta_{b}$. These burn angles, illustrated in Fig. 1, represent the crank angle interval between the start of combustion angle, $\theta_{\text {soc }}$, and $10 \%$ burned fuel mass and the interval between $10 \%$ and $90 \%$ burned fuel mass respectively. These burn angles are often referred to as the flame development angle and the rapid burn angle, see e.g. Heywood (1988).

Assuming that a burned mass fraction trace is specified, along with the total energy content of the injected fuel,

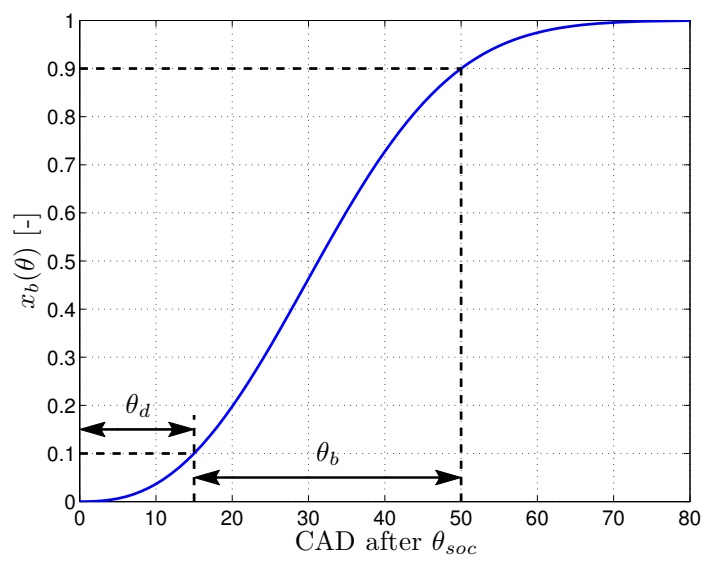

Fig. 1. Definition of the burn angles $\theta_{d}$ and $\theta_{b}$. Here, $\theta_{s o c}$ denotes the start of combustion angle.

the accumulated chemical energy release is given by (2). Hence, if (1) is rewritten as

$$
\frac{d p(\theta)}{d \theta}=\frac{\frac{d Q(\theta)}{d \theta}-\frac{\gamma}{\gamma-1} p(\theta) \frac{d V(\theta)}{d \theta}}{\frac{1}{\gamma-1} V(\theta)}
$$

and solved, the cylinder pressure trace generated by the specified combustion event can be calculated. Burned mass fraction traces are often specified using Vibe functions, introduced in Vibe (1956), for this purpose. This approach describes a model of the burned mass fraction as

$$
x_{b}(\theta)= \begin{cases}0 & , \quad \theta<\theta_{s o c} \\ 1-e^{-a\left(\frac{\theta-\theta_{s o c}}{\theta_{d}+\theta_{b}}\right)^{m+1}}, & \theta \geq \theta_{s o c}\end{cases}
$$

where $a$ and $m$ are referred to as the shape parameters of the Vibe function. These shape parameters can be expressed in terms of burn angles according to

$$
\begin{aligned}
& m=\frac{\ln (\ln (1-0.9)-\ln (1-0.1))}{\ln \left(\theta_{d}+\theta_{b}\right)-\ln \left(\theta_{d}\right)}-1 \\
& a=-\ln (1-0.1)\left(\frac{\theta_{d}+\theta_{b}}{\theta_{d}}\right)^{m+1}
\end{aligned}
$$

The described method for simulation of cylinder pressure traces is used to establish ideal properties of the proposed torque based combustion phasing estimation method that is introduced in the next section.

\section{TORQUE BASED COMBUSTION PHASING ESTIMATION}

This section gives a brief background to existing techniques for combustion phasing estimation based on crankshaft torque measurements. Also, an explanation of why $50 \%$ burned mass fraction cannot directly be calculated from such measurements is offered. After this, the new proposed combustion net torque method for torque based combustion phasing estimation is introduced. 


\subsection{Existing techniques}

There are two main issues when trying to estimate combustion phasing based on data from a crankshaft torque sensor. The first issue is that the measurements are not located within the cylinders but on the crankshaft. Hence, the measurements do not only reflect the individual combustions in each cylinder. Instead, these measurements include torque contributions from all cylinders as well as torque contributions resulting from the mechanical properties of the crankshaft. However, using a model of the crankshaft, it is possible, see e.g. Andersson and McKelvey (2004a), to recreate the cylinder individual torque contributions. This is referred to as cylinder separation and solves the sensor location issue.

The second issue is a result of the crank-slider mechanism that connects the straight-line motion of the piston to the rotary motion of the crankshaft. The gas pressure inside the cylinder produces gas torque, $T(\theta)$, through the piston and the crank-slider mechanism according to

$$
T(\theta)=p(\theta) A L(\theta)
$$

where $A$ is the piston area and $L(\theta)$ is the angle dependent crank lever. At top dead center (TDC) the crank lever is zero, causing numerical issues in the transformation from torque to pressure. Hence, $\theta_{x_{b} 50}$ cannot directly be calculated using (1) to (4) when these calculations are based on torque measurements. However, combustion phasing can still be calculated using the concept of torque ratio and the $50 \%$ torque ratio combustion phasing measure, both introduced in Andersson and McKelvey (2004b). This method uses a parameterized combustion model in order to overcome the numerical issue at TDC and the $50 \%$ torque ratio measure has proved to be highly correlated with $\theta_{x_{b} 50}$, see e.g. Andersson et al. (2008). The downside is that the fitting of the torque ratio model to data can make this method computationally expensive, especially for the complex combustion in a diesel engine.

\subsection{The combustion net torque method}

As an alternative to the torque ratio method for torque domain combustion phasing estimation, this work proposes the use of combustion net torque. The main motivation is to find an estimation method with low computational load that is well suited for online implementation. The combustion net torque, $T_{c}(\theta)$, is defined as

$$
T_{c}(\theta)=T(\theta)-T_{m}(\theta)
$$

where $T_{m}(\theta)$ denotes the torque produced by a corresponding motored engine cycle where no combustion occurs. Since the motored torque is subtracted from the total cylinder gas torque, the combustion net torque describes how the added net torque, generated by the combustion, evolves as a function of crank angle. An example of simulated fired and motored torque traces is shown in Fig. 2 along with the resulting combustion net torque trace.

Now, in order to use the combustion net torque to estimate $50 \%$ burned mass fraction it is necessary to establish a relation between the two. To do this, the location of
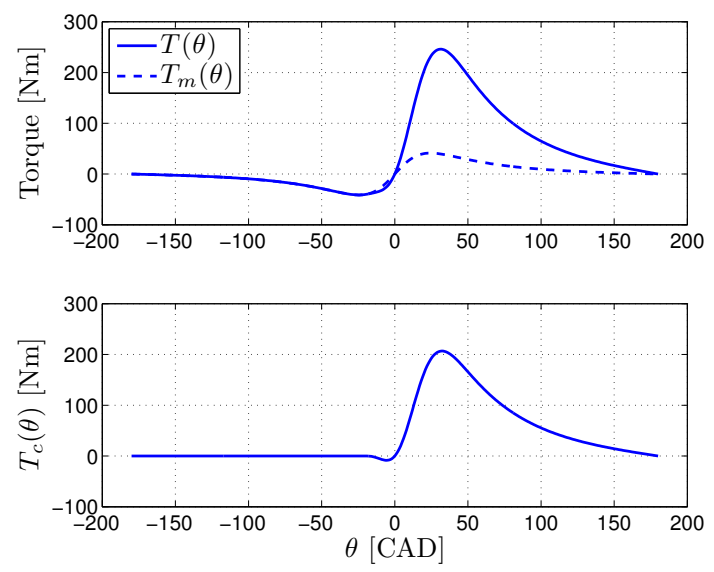

Fig. 2. An example of a combustion net torque trace (bottom) resulting from simulated fired and motored torque traces (top).

the midpoint on the positive flank of the combustion net torque trace, denoted $\theta_{T_{c} 50}$, is introduced and defined as

$$
T_{c}\left(\theta_{T_{c} 50}\right)=\frac{1}{2}\left(\min _{\theta} T_{c}(\theta)+\max _{\theta} T_{c}(\theta)\right)
$$

where $\theta_{T_{c} 50} \in\left[\theta_{\text {soc }}, \arg \max _{\theta} T_{c}(\theta)\right]$. The relation between $\theta_{x_{b} 50}$ and $\theta_{T_{c} 50}$ can now be expressed using an invertable so called linking function, $f_{\theta_{b}}\left(\theta_{x_{b} 50}\right)$, as

$$
\theta_{T_{c} 50}=f_{\theta_{b}}\left(\theta_{x_{b} 50}\right)
$$

Hence, an estimate, $\hat{\theta}_{x_{b} 50}$, of the $50 \%$ burned mass fraction position can be found by inverting (12). The properties of the proposed combustion net torque method will now be investigated in the remainder of this paper.

\section{SIMULATIONS}

Using the simulation model described in Section 2, with an engine geometry set to mimic the 2.4 liter spark ignited engine used in the experimental evaluation, the relation between $\theta_{x_{b} 50}$ and $\theta_{T_{c} 50}$ is studied for a large number of different burn angles and ignition positions. The results, summarized in Fig. 3, show that even if this relation is not affine, it is still strictly monotonic. Hence, a change in $\theta_{x_{b} 50}$ will always be detected in $\theta_{T_{c} 50}$ for combustions with identically shaped burn profiles. While the simulation results indicate that $\theta_{d}$ has a minimal influence on the link between the two measures, the results in Fig. 3 show a clear dependence on $\theta_{b}$, especially for late combustions. It is also noted that a change in engine geometry, e.g. compression ratio, influences the position of the curves in Fig. 3 but not their general shape. The results point out that the linking function for a given engine geometry needs to be parameterized in $\theta_{b}$, hence the function index in (12). In a real application however, $\theta_{b}$ may not be known. Estimation errors, $\tilde{\theta}_{x_{b} 50}$, defined as

$$
\tilde{\theta}_{x_{b} 50}=\hat{\theta}_{x_{b} 50}-\theta_{x_{b} 50}
$$

that arise if the influence of $\theta_{b}$ is neglected are therefore important to study. For instance, such estimation errors 


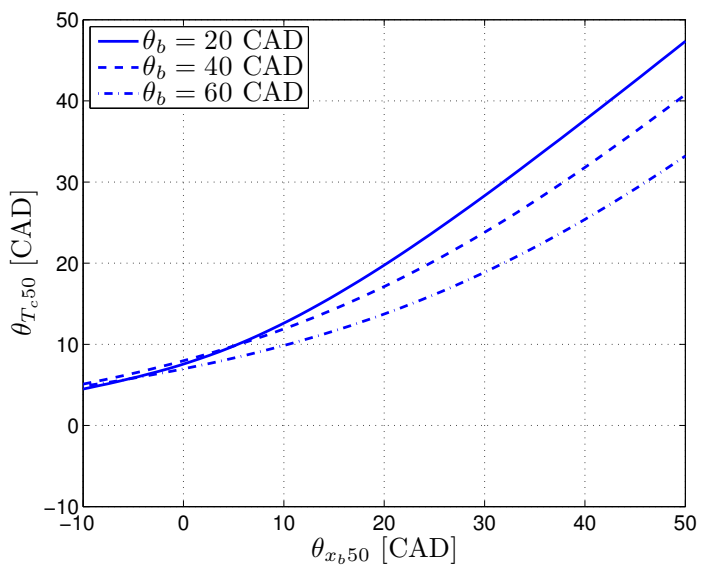

Fig. 3. Simulated relations between $\theta_{x_{b} 50}$ and $\theta_{T_{c} 50}$ for a given engine geometry and different values of $\theta_{b}$. Here, $\theta_{d}$ is $20 \mathrm{CAD}$.

could occur if an engine is running on fuel with unknown or varying properties that cause the burn profile to change. Summarized in Fig. 4 are results from an investigation of such estimation errors. Here, a linking function is fitted to simulated data in a nominal case, where $\theta_{d}$ is equal to $20 \mathrm{CAD}$ and $\theta_{b}$ is equal to $40 \mathrm{CAD}$, to represent this nominal data exactly. After this, new simulations are run where $\theta_{b}$ is altered, using perturbations denoted as $\Delta \theta_{b}$, to represent a change in burn rate. Based on these simulations, $\theta_{x_{b} 50}$ is estimated using the inverse of the nominal linking function and compared to the true $\theta_{x_{b} 50}$. As the linking function is no longer valid, due to the altered value of $\theta_{b}$, estimation errors are introduced. The results in Fig. 4 show how these estimation errors vary for different values of both $\Delta \theta_{b}$ and $\theta_{x_{b} 50}$. Here, a positive value of $\Delta \theta_{b}$ denotes an increased value of $\theta_{b}$, corresponding to a slower burning rate, and vice versa. The results indicate that the estimation errors grow as the combustion is retarded, consistent with the results in Fig. 3. For the studied values of $\Delta \theta_{b}, \pm 5$ and $\pm 10 \mathrm{CAD}$, the corresponding changes in the true $\theta_{x_{b} 50}$ value are approximately \pm 2 and \pm 4 CAD. This, together with (13) and the results in Fig. 4, concludes that the direction of a change in $\theta_{x_{b} 50}$ caused by a change in $\theta_{b}$ will be detected correctly even if an incorrect linking function is used.

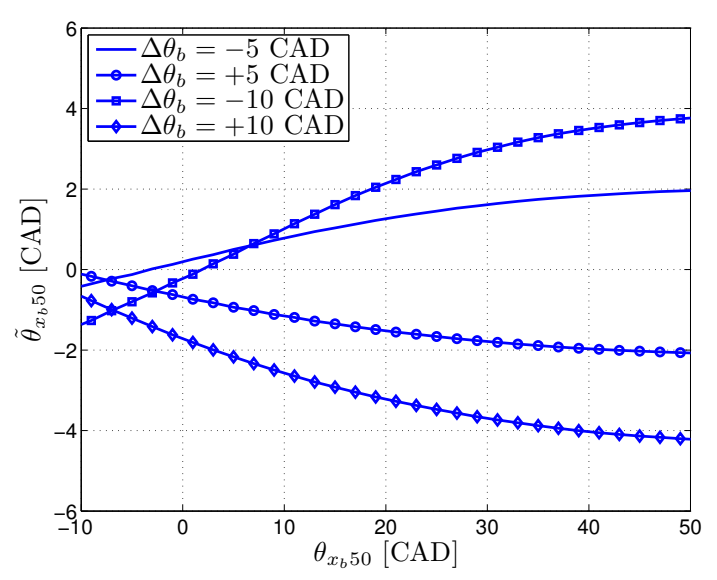

Fig. 4. An illustration of the errors in $\hat{\theta}_{x_{b} 50}$ that occur when the influence of $\theta_{b}$ is neglected. The $\Delta \theta_{b}$ values represent perturbations in $\theta_{b}$.
From a robustness point of view, it is also interesting to determine how the combustion net torque method is influenced by possible errors in the needed estimate of the motored torque in (10). A common method for the estimation of this torque is to assume that the cylinder gas undergoes adiabatic compression and expansion during the corresponding engine strokes. The motored cylinder pressure, $p_{m}(\theta)$, can thus be expressed as a polytropic process according to

$$
p_{m}(\theta)=p_{0}\left(\frac{V_{0}}{V(\theta)}\right)^{n}
$$

where $p_{0}$ and $V_{0}$ refer to cylinder pressure and cylinder volume at some datum point, e.g. at intake valve closing, and $n$ is the polytropic coefficient. The motored torque estimate is then formed in analogy with (9). Using this model for the motored torque means that it is necessary to estimate two parameters, $p_{0}$ and $n$. Methods for estimating these parameters vary in sofistication from using the pressure sensor in the engine's inlet manifold and prior knowledge about the polytropic coefficient to least-squares estimation techniques. Here, a small study is conducted in order to assess the robustness of the proposed method against errors in this estimation. For this purpose, simulations are performed using perturbations, described in Table 1, of nominal parameter values.

Table 1. Description of the parameter perturbations used to study the influence of motored torque estimation errors.

\begin{tabular}{ccccc} 
Parameter & Unit & Nominal value & $\Delta^{+}$ & $\Delta^{-}$ \\
\hline$p_{0}$ & bar & 0.26 & +0.1 & -0.1 \\
$n$ & - & 1.32 & +0.1 & -0.1 \\
\hline
\end{tabular}

The results from these simulations are depicted in Fig. 5. In the displayed simulation cases the values for $\theta_{d}$ and $\theta_{b}$ are $20 \mathrm{CAD}$ and $40 \mathrm{CAD}$ respectively. Generally, the shape of the curves in Fig. 5 are independent of the burn profile but the magnitude varies a little depending on $\theta_{b}$. Estimation errors tend to increase with increasing $\theta_{b}$ values and vice versa. Also, there is a clear indication that the estimation errors depend on the combustion phasing itself.
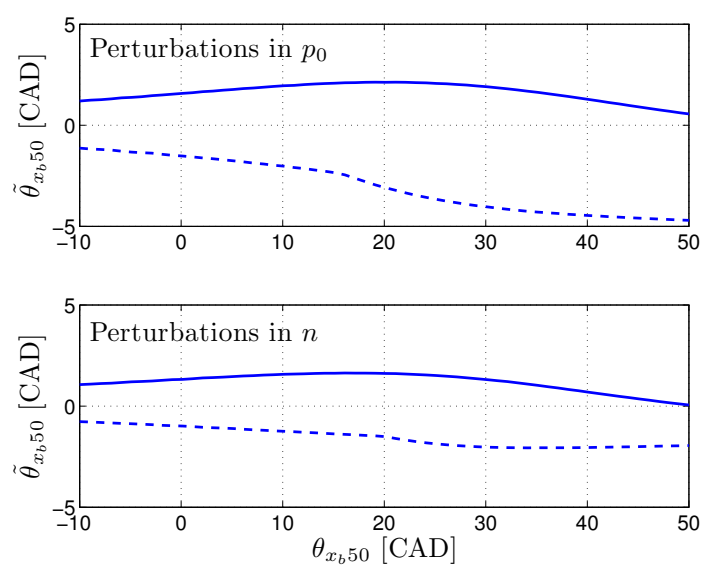

Fig. 5. Influence of motored torque parameter estimation errors on $\tilde{\theta}_{x_{b} 50}$. The solid lines indicate $\Delta^{+}$perturbations and the dashed lines indicate $\Delta^{-}$perturbations. 


\section{EXPERIMENTAL EVALUATION}

The work described in Section 4 establishes properties of the combustion net torque method using simulations. The purpose of the experiments in this section is to validate these properties and see if they hold true also for real engines. The experimental evaluation includes comparisons of the relation between $\theta_{x_{b} 50}$ and $\theta_{T_{c} 50}$ for two different engines and also an assessment of the $\theta_{x_{b} 50}$ estimation accuracy using the combustion net torque method.

\subsection{Experimental equipment}

The experimental evaluation is based on recorded cylinder pressure data from two different 5 -cylinder engines. The first is a spark ignited engine with a total displacement of 2.4 liters and a compression ratio of 10.3 and the second is a 2.4 liter diesel engine with a common-rail injection system and a compression ratio of 17.3. Both engines are, to a large extent, standard production engines manufactured by Volvo Car Corporation. All cylinders in both engines were, however, instrumented with pressure sensors, sampled with a resolution of $1 \mathrm{CAD}$.

The spark-ignited engine experiments were carried out at an engine speed of 2000 revolutions per minute (RPM) and a maximum engine load of approximately 2 bar brake mean effective pressure (BMEP). The experiments using the diesel engine were carried out at four different operating points, including engine speeds of 1000 and 2000 RPM and maximum engine loads of approximately 1 and 4 bar BMEP. In the experiments, the combustion phasing was altered using spark timing for the spark ignited engine and injection timing for the diesel engine.

\subsection{Results}

The data in Fig. 6 and Fig. 7 provide a summary of the conducted engine experiments. Fig. 6 illustrates the relation between $\theta_{x_{b} 50}$ and $\theta_{T_{c} 50}$ for 3000 engine cycles from the spark ignited experimental engine and Fig. 7 shows the corresponding relation for 7600 cycles across all four operating points for the experimental diesel engine. It is tempting to relate these results directly to the simulation results in Fig. 3, but there is a fundamental difference between these plots. For the real combustion process there is a

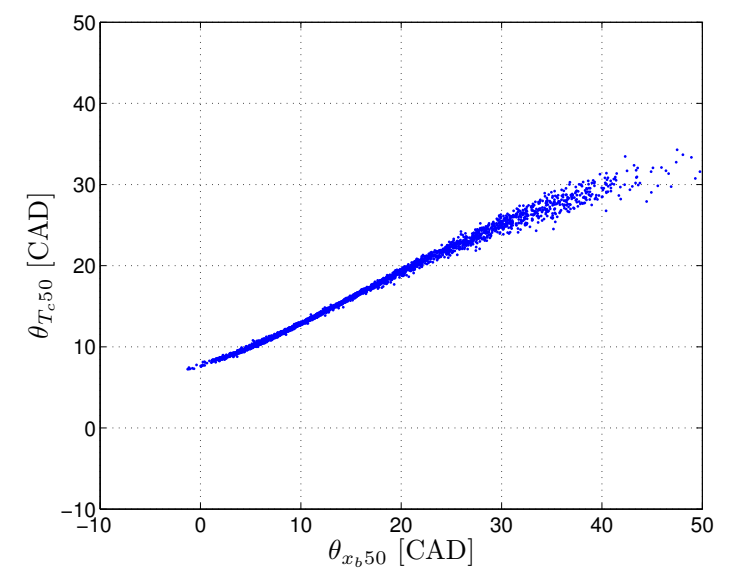

Fig. 6. The relation between $\theta_{x b 50}$ and $\theta_{T_{c} 50}$ for the spark ignited experimental engine.

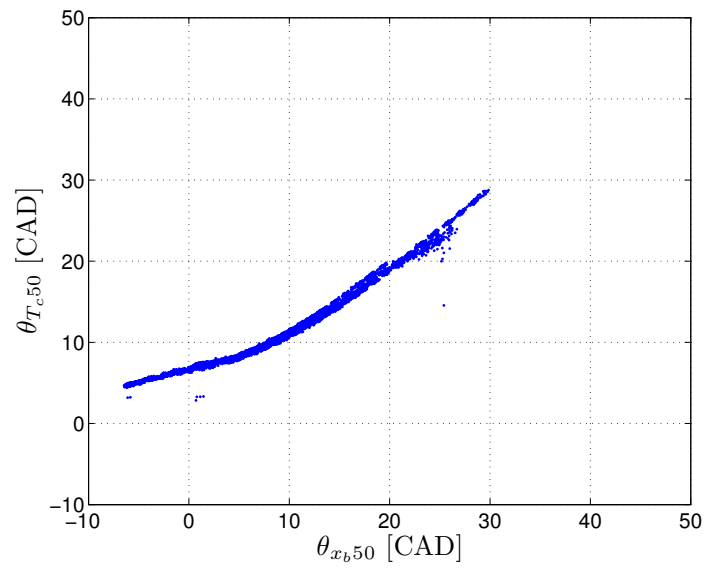

Fig. 7. The relation between $\theta_{x_{b} 50}$ and $\theta_{T_{c} 50}$ for the experimental diesel engine.

dependence between $\theta_{x_{b} 50}$ and $\theta_{b}$ and as the combustion is retarded the rapid burn angle increases. Hence, the shape of the experimental relation cannot directly be compared to the shape of the simulated relation as the rapid burn angle varies in the experimental data. The reason why the data from the spark ignited engine is less curved than for the diesel engine data is that $\theta_{b}$ varies more over the $\theta_{x_{b} 50}$ range than it does for the diesel engine. The significant compression ratio difference between the two engines also influences the results. However, if the comparison is restricted to early combustions, where the simulations show a small burn angle dependence, the agreement between the experimental data and the simulations is good.

In spite of the discussed difference between the simulations and the experiments, the experimental relation between $\theta_{x_{b} 50}$ and $\theta_{T_{c} 50}$ can still be described by fitting linking functions to the data. The difference is that, in this case, the linking functions will have an implicit dependence on $\theta_{b}$, instead of an explicit dependence as seen before. A single linking function will therefore only be valid within an engine operating range where this implicit dependence does not change. The small spread across all operating points for the diesel data in Fig. 7 indicates such a constant dependence for that specific operating range.

To assess how accurately the combustion net torque method is able to estimate $50 \%$ burned mass fraction, within the operating range of the experiments, two linking functions are used, one for the spark ignited engine data and one for the data from the diesel engine. For this purpose, the two data sets are divided into two parts each, one used for estimation and one used for validation. Here, these linking functions were chosen as two fourth order polynomials. The results are depicted in Fig. 8, for the spark ignited engine, and Fig. 9, for the diesel engine. These results show that the combustion net torque method is able to estimate $\theta_{x_{b} 50}$ with high accuracy, especially for the crank angle region around 5-15 CAD after TDC where $\theta_{x_{b} 50}$ normally is positioned for engine efficiency reasons. Overall statistics of the estimation accuracy for the spark ignited engine data show an ideal estimation bias of $0.04 \mathrm{CAD}$ and a standard deviation of $0.9 \mathrm{CAD}$. The corresponding statistics for the diesel engine data show a bias of 0.01 CAD and a standard deviation of 0.6 CAD. It is, however, important to stress that more experiments 


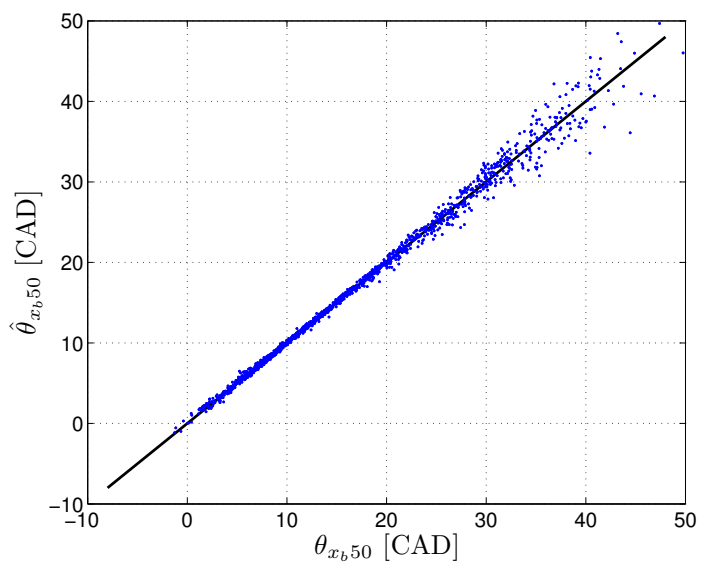

Fig. 8. Combustion phasing estimation using the combustion net torque method for the spark ignited engine.

need to be conducted in order to give a complete assessment of the proposed method. These experiments should cover the entire operating range of the engines and also use e.g. different fuel qualities in order to further understand the influence of varying burn rates on estimation accuracy.

Finally, in order to connect back to the motivation for the combustion net torque method, the computational demand of estimating $50 \%$ burned mass fraction using this method is compared to that of using the torque ratio method. It turns out that, for the spark ignited engine, the gain of using combustion net torque is around a factor of 40. For the diesel engine, needing a more complex torque ratio model, the gain is around a factor of 250 .

\section{CONCLUSIONS}

This paper proposes a new method, called the combustion net torque method, for estimating combustion phasing based on crankshaft torque measurements. The motivation for the work is the need for a computationally efficient combustion phasing estimation method for real-time implementation in a torque based closed-loop combustion phasing control system. In this work, the ideal relation between the combustion net torque method and $50 \%$ burned mass fraction, using torque calculated from cylinder pressure, is established and the properties of the estimation method are studied. The investigations are based on both simulations and experimental data, collected from one spark ignited and one compression ignited engine.

The simulation results show that there is a strong, but not affine, link between the combustion net torque and the location of $50 \%$ burned mass fraction. The use of so called linking functions enables $50 \%$ burned mass fraction to be estimated based on the combustion net torque. These linking functions turn out to be dependent on the engine geometry and the burn rate of the combustion. An analysis of the impact on the estimation accuracy if this dependence is neglected is also performed. A neglected burn rate change of $10 \mathrm{CAD}$ can cause estimation errors of up to 4 CAD. Also, the influence of motored torque estimation quality is studied and shown to be limited.

The use of experimental cylinder pressure data shows that the proposed method's ideal estimates have a bias of 0.04 CAD for the spark ignited engine and 0.01 CAD

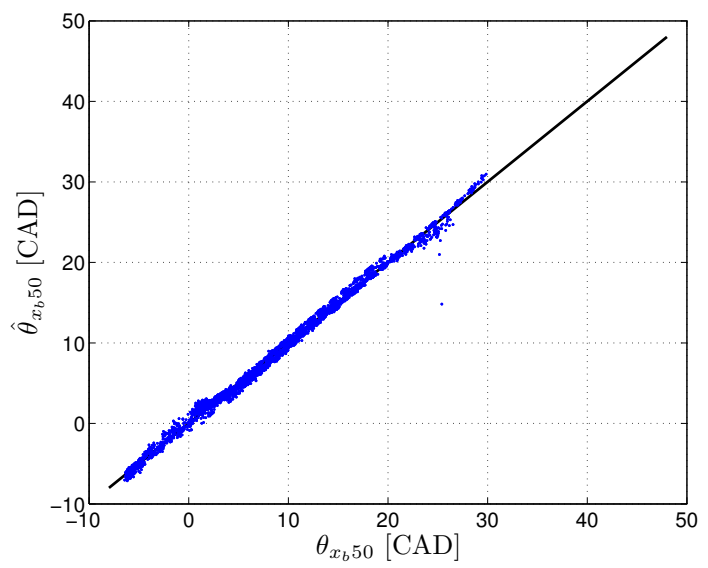

Fig. 9. Combustion phasing estimation accuracy using the combustion net torque method for the diesel engine.

for the diesel engine. Also, results indicate that the computational load using the combustion net torque method decrease with as much as a factor of 250 compared to existing torque domain estimation methods. However, further experiments, spanning a larger engine operating range and varying combustion characteristics, are needed to fully understand the properties of the proposed method and its relation to $50 \%$ burned mass fraction. Currently, work dealing with these issues, as well as an extension of the combustion net torque method that eliminates the influence of burn angles in the estimation of $50 \%$ burned mass fraction, is being performed by the authors.

\section{REFERENCES}

Andersson, I. and McKelvey, T. (2004a). A System Inversion Approach on a Crankshaft of an Internal Combustion Engine. In Proceedings of the $43 \mathrm{rd}$ IEEE Conference on Decision and Control, 5449-5454.

Andersson, I. and McKelvey, T. (2004b). Torque Ratio Concept for Combustion Phasing Detection of a Spark Ignited Engine. In Proceedings of the 43rd IEEE Conference on Decision and Control, 1703-1708.

Andersson, I., McKelvey, T., and Thor, M. (2008). Evaluation of a Closed Loop Spark Advance Controller Based on a Torque Sensor. SAE Technical Paper Series 2008$01-098 \%$.

Gatowski, J.A., Balles, E.N., Chun, K.M., Nelson, F.E., Ekchian, J.A., and Heywood, J.B. (1984). Heat Release Analysis of Engine Pressure Data. SAE Technical Paper Series 841359.

Heywood, J.B. (1988). Internal Combustion Engine Fundamentals. McGraw-Hill.

Matekunas, F.A. (1986). Engine combustion control with ignition timing by pressure ratio management. US Patent 4,622,939.

Rassweiler, G.M. and Withrow, L. (1938). Motion Pictures of Engine Flames Correlated with Pressure Cards. SAE Journal of Transactions, 42(5), 185-204.

Thor, M., Andersson, I., and McKelvey, T. (2009). Parameterized Diesel Engine Heat Release Modeling for Combustion Phasing Analysis. SAE Technical Paper Series 2009-01-0368.

Vibe, I.I. (1956). Semi-empirical expression for combustion rate in engines. In Proceedings of a Conference on Piston Engines, 185-191. 